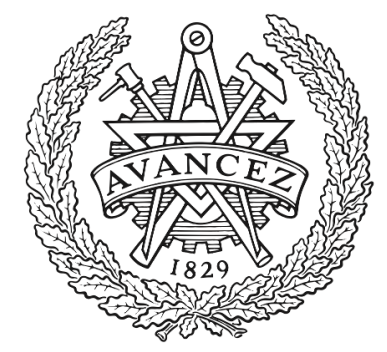

\title{
CHALMERS
}

UNIVERSITY OF TECHNOLOGY

\section{Analysis and Estimation of the Maximum Switch Current during Battery System Reconfiguration}

Downloaded from: https://research.chalmers.se, 2023-04-26 14:30 UTC

Citation for the original published paper (version of record):

Han, W., Kersten, A., Zou, C. et al (2022). Analysis and Estimation of the Maximum Switch Current during Battery System Reconfiguration. IEEE Transactions on Industrial Electronics, 69(6): 5931-5941. http://dx.doi.org/10.1109/TIE.2021.3091923

N.B. When citing this work, cite the original published paper.

(O2022 IEEE. Personal use of this material is permitted.

However, permission to reprint/republish this material for advertising or promotional purposes 


\title{
Analysis and Estimation of the Maximum Switch Current during Battery System Reconfiguration
}

\author{
Weiji Han, Member, IEEE, Anton Kersten, Student Member, IEEE, Changfu Zou, Member, IEEE, \\ Torsten Wik, Xiaoliang Huang, Member, IEEE, Guangzhong Dong, Member, IEEE
}

\begin{abstract}
Batteries are interconnected in series and/or parallel to meet wide-range power or energy demands in various industrial applications. To pursue the benefits of multiple connection structures in one system, reconfigurable battery systems (RBSs) have recently emerged for safe and efficient operation, extended energy storage and delivery, etc. Switches are the essential elements to enable the battery system reconfiguration, but selecting appropriate switches for RBS designs has not been systematically investigated. To bridge this gap, analytical expressions are derived in this paper to estimate the maximum switch current and its upper limit to facilitate the selection of RBS switches. An RBS prototype based on H-bridges is set up and experimental results verify the effectiveness and advantage of the proposed estimation method. These analytical expressions, relying only on resistances of batteries and switches, are readily applicable to practical RBS design and much more efficient than conducting numerous circuit experiments, simulation tests, or circuit analyses, especially for large-scale systems. Moreover, the analysis framework and estimation method proposed for seriesparallel mutual conversion can be adaptively extended to other complex system reconfigurations to facilitate various RBS designs.
\end{abstract}

Index Terms-Battery system, estimation, parallel connection, reconfiguration, switch current analysis.

\section{INTRODUCTION}

Battery energy storage and delivery have been playing an important role in a variety of industrial applications, such as transportation electrification and integration of wind and solar energy to power grids. The functionality and performance of a multi-battery system depend not only on the batteries applied but also on the way they are interconnected, i.e., the system configuration. As compared to the research and industrial efforts devoted to developing various high-performance batteries within a fixed configuration, far less attention has been paid to investigating the influence of dynamic system configurations. However, it has been revealed that appropriately manipulating

Manuscript received Month $\mathrm{xx}, 2 \mathrm{xxx}$; revised Month $\mathrm{xx}$, xxxx; accepted Month $x, x x x x$. This work was supported in part by Chalmers Transport Area of Advance and in part by Mistra Innovation under project MI23-19.03. (Corresponding author: Guangzhong Dong.)

W. Han, A. Kersten, C. Zou, T. Wik, and X. Huang are with the Department of Electrical Engineering, Chalmers University of Technology, Gothenburg 41296, Sweden (e-mail: weiji.han@chalmers.se, kersten@chalmers.se, changfu.zou@chalmers.se, tw@chalmers.se, xiaoliang.huang@chalmers.se).

G. Dong is with the School of Mechanical Engineering and Automation, Harbin Institute of Technology, Shenzhen, Guangdong 518055, China (e-mail: gzdong@ieee.org). the system configuration can bring about a number of potential benefits [1], in terms of, e.g., enhanced fault tolerance [2], improved charge balance [3], [4], extended energy delivery [5], programmable terminal voltages [6], as well as hybrid use of batteries of different ages, types, and even chemistries [7]. In view of this, reconfigurable battery systems (RBSs) have been recognized as a promising direction to advanced battery management and control [1].

To set up an RBS, switches are introduced and connected between battery cells/modules/packs. As a result, unlike a conventional battery system of a fixed configuration, an RBS can operate in different interconvertible connection structures by controlling the states of switches. Various RBS topology designs and hardware implementations have been extensively reviewed in [1], [8], [9]. For instance, as shown in Fig. 2 and Table 1 in [1], a total of 11 emerging RBS topology designs have been compared and analyzed. These RBSs were designed for enhanced fault tolerance, customized terminal voltage range, charge and temperature balance, extended energy delivery, hybrid application of heterogeneous batteries, etc. To pursue these benefits, RBS management principles as well as control and optimization algorithms have been comprehensively discussed in [1]. On the other hand, to date, several challenges still need to be addressed in the RBS hardware design, system modeling, algorithm development, switch operations, scalability, etc.

During the hardware design of RBSs, to implement various system configurations with guaranteed safety under any encountered condition, RBS components should be appropriately selected based on their operating limits. In addition to choosing proper batteries and wires as required in all battery system designs, particular attention needs to be paid to the switch selection in the RBS hardware design. Due to the heating effect caused by resistive losses in internal components of switches, the continuous and various pulsed switch currents are bounded by certain limits specified by manufacturers. For instance, the switch in [10] can conduct currents up to 5.2 A for continuous operation and up to $21 \mathrm{~A}$ for a 160 microsecond pulse. When an RBS is reconfigured, e.g., from series to parallel, switch currents can dramatically change and potentially render substantial spikes (as will be illustrated in Section IV). While such current spikes typically fade very quickly, an inappropriately selected switch might suffer from overcurrent for certain pulse duration, causing progressive damage to the switch often with little noticeable temperature rise before failure [11]. However, the maximum RBS switch 
currents, especially during the system reconfiguration, have not been systematically studied in the literature.

To bridge this research gap, this paper will focus on analyzing and estimating the maximum switch currents for the switch selection in a typical RBS. The evolution of switch currents during battery system reconfiguration depends on the battery terminal voltages as well as the original and new system connection structures. Given the requested system reconfiguration, e.g., from series to parallel, a variety of possible battery voltages as well as their permutations need to be considered for quantifying the maximum switch current magnitudes. Conducting an exhaustive search among all possible cases by circuit experiments or model-based simulations would be too inefficient in energy and time, especially for large-scale RBSs. Moreover, instead of investigating only one fixed configuration in conventional battery systems, all desired configurations of the RBS along with their mutual conversions need to be comprehensively assessed. The maximum switch currents under different RBS configurations need to be well coordinated to ensure safe operation.

To address these challenges, particularly during the system reconfiguration, analytical expressions for directly evaluating the maximum switch current as well as its upper limit are first derived. As the major contribution of this paper, the proposed estimation equations are readily applicable, and, hence, much more energy- and time-efficient than exhaustively testing various battery voltage permutations by experiments or simulations. The estimated maximum switch currents and upper limits provide a ground for selecting appropriate switches for RBSs as well as checking the feasibility of the requested reconfiguration. Moreover, while the analysis and estimation methods are proposed for the reconfiguration between basic series and parallel connections, they are potentially extendable to more complicated reconfiguration operations which, in essence, are still composed of these two basic connection patterns, so as to cater for various RBS designs.

In the remainder of this paper, the following investigations will be subsequently conducted, significantly extending our preliminary work in [12]. Specifically, a typical RBS circuit design, allowing flexible conversion between series and parallel battery connections, will be mathematically modeled in Section II. Based on this model along with reasonable assumptions, the maximum switch currents under various system configurations and operating conditions will be analyzed and estimated, and their upper limits will then be derived in Section III. The effectiveness of the proposed estimation method will be verified by circuit experiments on an RBS prototype designed based on H-bridge converters in Section IV. Finally, conclusions and future work will be presented in Section V.

\section{SYSTEM MODELING OF RECONFIGURATION CIRCUIT DESIGN}

\section{A. Circuit design for flexible series and parallel connec- tions}

In order to realize different configurations in a battery system, switches are commonly inserted between battery

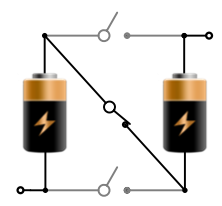

(a)

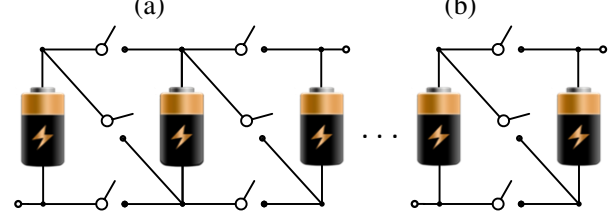

(c)

Fig. 1. Arrangement and working principle of an RBS allowing flexible conversion between series and parallel battery connections. (a) Series connection. (b) Parallel connection. (c) RBS design for multiple cells.

cells/modules/packs to activate or terminate their electrical connections. Depending on the number of switches around each battery and the battery-switch connection pattern, various RBS designs have been proposed recently and they were reviewed in [1], [8], and [9]. In all these RBS designs, batteries are locally connected in either series or parallel. In view of this, a typical RBS design enabling the mutual conversion between these two basic connection patterns is studied in this section.

Consider interconvertible series and parallel connections of only two battery cells at first. Clearly, the series and parallel connections of two cells should be exclusive to avoid any short circuit. Thus, when reconfiguring two parallelconnected cells to series connection, two switches, referred to as parallel switches, are needed to deactivate the original parallel connection (See Fig. 1 (a)). Then, to convert the series configuration back to a parallel connection, another switch, referred to as the series switch, needs to be inserted to cut off the series conduction path (See Fig. 1 (b)). Therefore, at least three switches are required to enable the flexible conversion between series and parallel connections of any two adjacent battery cells. Finally, the two-cell system can be scaled up to incorporate more cells, as shown in Fig. 1 (c).

Through flexible series-parallel reconfiguration in such an RBS design, the system can provide a wide range of terminal voltage [1], [13]-[15], which can facilitate the operation of various EV components with different voltage levels and waveforms. For some fast transient scenarios requiring high switching frequency, multiple battery cells/modules need to be reconfigured as simultaneously as possible [14]. In addition, any fully charged/discharged or faulty cell in a seriesconnected battery string can be individually isolated on the fly to achieve continuous and robust system operation. This reconfigurable topology was also applied to supercapacitors in [16] to improve the cycling efficiency and capacity utilization.

\section{B. Equivalent circuit diagrams}

During the hardware design, RBS components should be selected to allow for various connection structures and working conditions. To perform various tests, a well-established circuit model would be an efficient tool. Not only all components, such as battery cells, switches, and wires, but also their 


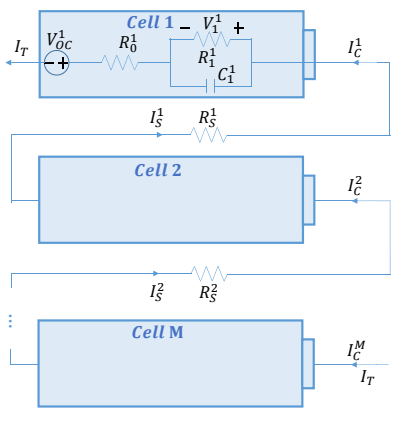

(a)

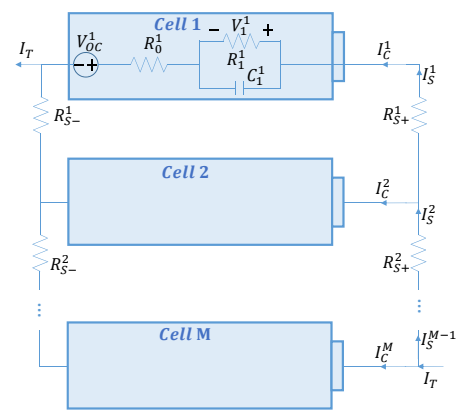

(b)
Fig. 2. Equivalent circuit diagrams of two basic configurations of the RBS in Fig. 1. (a) Series connection. (b) Parallel connection.

possible connection structures need to be modeled. Through different switch operations in the RBS in Fig. 1, $M$ battery cells can get connected in one of the two basic configurations, either series or parallel, and their equivalent circuit diagrams are illustrated in Fig. 2 (a) and (b), respectively.

To model battery cells, equivalent circuit models (ECMs) are deployed since they can directly describe the electrical characteristics of batteries at an acceptable computational cost. It can be seen from Fig. 2 that a typical battery cell ECM is composed of a voltage source indicating its open circuit voltage (OCV) denoted by $V_{O C}^{m}, m=1, \ldots, M$, a resistor characterizing its internal resistance $R_{0}^{m}$, and $N \geq 1$ parallel resistor-capacitor (RC) pairs to describe the dynamic battery behavior such as the diffusion effect. The $n$-th RC pair in the $m$-th cell's ECM consists of a resistor $R_{n}^{m}$ connected in parallel with a capacitor $C_{n}^{m}$, and its voltage is denoted by $V_{n}^{m}, n=1, \ldots, N$. For clear illustration, $N=1$ is set for all cells in Fig. 2.

The switch modeling depends on the type of switches to be applied. MOSFET switches outperform mechanical ones in terms of much lower power demand for actuation and better synchronization performance for parallel operation [17]. Thus, MOSFET switches are employed here and modeled by its on-resistance, defined by the total resistance on the conductive path when the switch is turned on [18]-[20]. Specifically, as shown in Fig. 2, the series switch's resistance is denoted by $R_{S}^{m}$, and the resistances of parallel switches connecting the positive and negative battery terminals are denoted by $R_{S+}^{m}$ and $R_{S-}^{m}$, respectively. In addition, since the resistances of connection wires or contacts can be easily incorporated into the resistances of nearby series-connected battery cells or switches, they are not explicitly shown in Fig. 2.

\section{Mathematical modeling}

For the series battery connection in Fig. 2 (a), clearly, all battery and switch currents are always equal to the RBS's total current $I_{T}$. Thus, batteries and switches should be capable of conducting the maximum charging and discharging currents of the RBS. When the system is reconfigured to the parallel connection as shown in Fig. 2 (b), however, the maximum switch currents are not so straightforward anymore due to the dynamic distribution of the total current to all parallel cells. Then, a mathematical model is formulated for analyzing the switch current evolution during the series-to-parallel reconfig- uration and the following parallel operation. To simplify the notation, all time indices are omitted in this section.

During the RBS operation, the time-varying battery OCVs and RC pair voltages are selected as state variables to compose the state vector $x=\left[V_{O C}^{1}, V_{1}^{1}, \ldots, V_{N}^{1}, \ldots, V_{O C}^{M}, V_{1}^{M}, \ldots, V_{N}^{M}\right]^{T}$, $x \in \mathbb{R}^{M(N+1)}$, such that

$$
\begin{aligned}
& \dot{V}_{O C}^{m}=\frac{\mathrm{d} V_{O C}^{m}}{\mathrm{~d} z_{m}} \dot{z}_{m}=k_{V S} \frac{\dot{Q}_{m}}{Q_{C}}=\frac{k_{V S}}{Q_{C}} I_{C}^{m}, \\
& \dot{V}_{n}^{m}=\frac{1}{C_{n}^{m}}\left(I_{C}^{m}-\frac{V_{n}^{m}}{R_{n}^{m}}\right)=\frac{-1}{R_{n}^{m} C_{n}^{m}} V_{n}^{m}+\frac{1}{C_{n}^{m}} I_{C}^{m}, \\
& n=1, \ldots, N .
\end{aligned}
$$

In (1), $z_{m}$ is the $m$-th cell's state of charge (SoC), defined by the ratio of its present amount of charge $Q_{m}$ and its charge capacity, and $I_{C}^{m}$ denotes the $m$-th cell's current, positive for charging. Assume all cells have the same charge capacity $Q_{C}$ and share the same OCV-SoC curve. Then, $k_{V S}\left(z_{m}\right)$ denotes the slope of the OCV-SoC curve, dependent on the real-time SoC $z_{m}$ or OCV of each cell, and, hence, the system state $x$.

Denote the cell current vector by $I_{C}=\left[I_{C}^{1}, \ldots, I_{C}^{M}\right]^{T}$, then the state-space representation of battery cells can be generalized from (1) and (2) as

$$
\dot{x}=A_{C} x+B_{C} I_{C} .
$$

The system evolution represented by (3) is driven by all cell currents in $I_{C}$, which cannot be individually assigned during the parallel operation due to the dynamic parallel current distribution. Alternatively, the total current of the RBS, $I_{T}$, can be directly controlled. Thus, the input $I_{C}$ in (3) will be expressed in terms of the total system current $I_{T}$ as follows.

Applying Kirchhoff's voltage law (KVL) to the loop composed of the $m$-th and $(m+1)$-th cells and two parallel switches in Fig. 2 (b), $m=1,2, \ldots, M-1$, we have

$$
\begin{aligned}
& -V_{O C}^{m}-I_{C}^{m} R_{0}^{m}-\sum_{n=1}^{N} V_{n}^{m}-I_{S}^{m} R_{S+}^{m}+\sum_{n=1}^{N} V_{n}^{m+1} \\
& +I_{C}^{m+1} R_{0}^{m+1}+V_{O C}^{m+1}+\left(I_{T}-I_{S}^{m}\right) R_{S-}^{m}=0,
\end{aligned}
$$

where $I_{S}^{m}$ denotes the current through the parallel switch connecting the positive terminals of the $m$-th and $(m+1)$-th cells. Then, applying Kirchhoff's current law (KCL) to the positive terminals of $M$ cells, we have

$$
\begin{aligned}
& I_{S}^{m}=\sum_{k=1}^{m} I_{C}^{k}, m=1,2, \ldots, M-1, \\
& I_{T}=I_{S}^{M-1}+I_{C}^{M}=\sum_{m=1}^{M} I_{C}^{m} .
\end{aligned}
$$

Combining and reorganizing (6) and the $M-1$ equations in (4) with $I_{S}^{m}$ substituted by (5) leads to

$$
E x+F I_{C}=G I_{T} \Rightarrow I_{C}=-F^{-1} E x+F^{-1} G I_{T} .
$$

Then, based on (3) and (7), the state-space representation for the RBS parallel operation driven by $I_{T}$ is

$$
\dot{x}=\left(A_{C}-B_{C} F^{-1} E\right) x+B_{C} F^{-1} G I_{T} \triangleq A x+B I_{T} .
$$


Finally, according to (5), the switch current vector, denoted by $I_{S}=\left[I_{S}^{1}, \ldots, I_{S}^{M-1}\right]^{T}$, can be represented by the cell current vector $I_{C}$ through a transition matrix, denoted by $T_{S C}$, and based on (7),

$$
\begin{aligned}
I_{S} & =T_{S C} I_{C} \\
& =-T_{S C} F^{-1} E x+T_{S C} F^{-1} G I_{T} \triangleq C_{S} x+D_{S} I_{T} .
\end{aligned}
$$

The involved matrices $A_{C}, B_{C}, E, F, G$, and $T_{S C}$ in (3), (7), (8), and (9) can be derived based on (1), (2), (4), (5), and (6), and their entries will not be presented here for brevity. Note that, $B_{C}, A$, and $B$ in (8) are not constant since $B_{C}$ has entries, i.e., $\frac{k_{V S}}{Q_{C}}$, dependent on the evolution of cell SoCs or OCVs. Thus, (8) represents a time-varying system.

This system model (8) along with the output equation (9) can be used to simulate and analyze the switch current evolution during the RBS series-to-parallel operation. However, since a lot of battery states and input currents are possible, it is too inefficient to test all possible cases for identifying the maximum switch current. To accomplish this, direct analytical expressions for the maximum RBS switch currents as well as their upper limits will be derived.

\section{ANALYSIS AND ESTIMATION OF THE MAXIMUM SWITCH CURRENT}

As discussed previously, once the system is reconfigured to the series connection, the maximum series switch current can be directly determined by the maximum external current of the RBS. Thus, the maximum switch current analysis will be mainly focused on the series-to-parallel reconfiguration and the following parallel operation based on the developed model in Section II. Consider new cells and switches sharing identical parameter values, i.e., for $\forall m$ in Fig. 2 (b),

$$
R_{0}^{m}=R_{C}, R_{n}^{m}=R_{n}, C_{n}^{m}=C_{n}, R_{S+}^{m}=R_{S-}^{m}=R_{S} .
$$

\section{A. Current analysis for the series-to-parallel reconfigura- tion}

Consider battery cells first operating in series and then being reconfigured to parallel connection. During the series operation, since all cells always share the same charging or discharging current, the SoC difference between battery cells remains unchanged, and, accordingly, the cell OCVs cannot get balanced. Other entries in the state vector, i.e., RC pair voltages, however, can be shown to gradually get equalized during the series operation as follows.

Based on (2) and (10), for each RC pair in each cell,

$$
V_{n}(t)=e^{-\frac{t}{R_{n} C_{n}}} V_{n}(0)+\int_{0}^{t} e^{-\frac{t-\tau}{R_{n} C_{n}}} \frac{I_{T}(\tau)}{C_{n}} d \tau,
$$

in which the first term always decays over time and will approach zero when the series operation time is much longer than the time constant $\tau_{n}=R_{n} C_{n}$, typically only seconds. Thus, as the series operation goes on, each cell's $V_{n}(t)$ is gradually dominated by the second term in (11), which is always identical for all series-connected cells since they share the same total current $I_{T}(t)$ and parameter values of $R_{n}, C_{n}$ based on (10). In other words, after a sufficiently long period of series operation, all battery cells will have identical RC pair voltages, and, hence, the cell state imbalance will only depend on cell OCVs.

Once the system is reconfigured to parallel operation, the switch current $I_{S}$ in (9) can be divided into the zero-input and zero-state terms, i.e.,

$$
\begin{aligned}
& I_{S z i} \triangleq C_{S} x, \\
& I_{S z s} \triangleq D_{S} I_{T} .
\end{aligned}
$$

Then, the maximum switch current magnitude satisfies

$$
\begin{aligned}
\max _{m, t}\left|I_{S}^{m}(t)\right| & =\max _{m, t}\left|I_{S z i}^{m}(t)+I_{S z s}^{m}(t)\right| \\
& \leq \max _{m, t}\left(\left|I_{S z i}^{m}(t)\right|+\left|I_{S z s}^{m}(t)\right|\right) \\
& \leq \max _{m, t}\left|I_{S z i}^{m}(t)\right|+\max _{m, t}\left|I_{S z s}^{m}(t)\right|,
\end{aligned}
$$

where the superscript $m$ indicates the $m$-th entry in vectors $I_{S}, I_{S z i}$, and $I_{S z s}$.

Based on (13), the zero-state term in (14) can be estimated by

$$
\begin{gathered}
\max _{m, t}\left|I_{S z s}^{m}(t)\right|=\max _{m, t}\left|D_{S}^{m} I_{T}(t)\right|=\max _{m, t}\left(\left|D_{S}^{m}\right|\left|I_{T}(t)\right|\right) \\
=\max _{m}\left|D_{S}^{m}\right| \max _{t}\left|I_{T}(t)\right|=\max _{m}\left|D_{S}^{m}\right| I_{T}^{u b} \triangleq\left|I_{S z s}^{\max }\right|
\end{gathered}
$$

where $D_{S}^{m}$ denotes the $m$-th entry of the vector $D_{S}$, which is determined by all cell and switch resistances, and, thus, assumed constant over time. Then, $\max _{m}\left|D_{S}^{m}\right|$ can be easily identified based on the pre-calculated $D_{S}$. Besides, $I_{T}^{u b}$ is the pre-specified upper bound for the magnitude of the total external current. Thus, $\left|I_{S z s}^{\max }\right|$ can be directly calculated and the following analysis will be mainly concentrated on estimating the remaining zero-input term in (14).

Given zero external current during the parallel operation, if the cell terminal voltages are initially different, they will gradually get balanced with each other and cause decreasing balancing currents through switches over time. Then, the maximum switch current magnitude should be observed at the beginning of the parallel operation, and the corresponding time instant is denoted by $t_{p}$. To illustrate this, based on the developed model (8) with $I_{T}=0$, the parallel operation of a 4 -cell RBS is simulated in Fig. 3 with $t_{p}=0$. As one can see, the maximum switch current's magnitude (marked by the blue solid curve) decreases over time. Thus, the zero-input term in (14) becomes

$$
\max _{m, t}\left|I_{S z i}^{m}(t)\right|=\max _{m}\left|I_{S z i}^{m}\left(t_{p}\right)\right| .
$$

To estimate this current magnitude, the initial balancing currents during the parallel operation will be intensively analyzed. For brevity, all the following variables and equations in this section will be presented at $t=t_{p}$ if not particularly specified.

\section{B. Initial switch current analysis during the parallel oper- ation without external charging/discharging current}

As discussed at the beginning of Subsection III-A, during the series operation, RC pair voltages can gradually get equalized for all cells but the cell OCV imbalance cannot be alleviated. When the system is reconfigured from series 


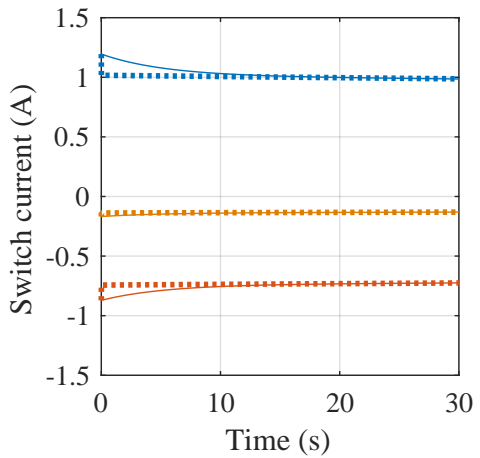

Fig. 3. The switch current evolution during the parallel operation of a 4cell RBS based on the complete model represented by (8) (solid curves) and that based on the model without RC pairs (dot curves).

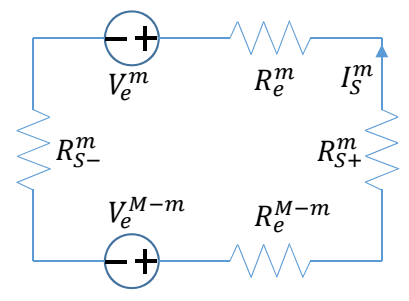

Fig. 4. Equivalent circuit of two two-terminal networks connected through parallel switches.

to parallel at $t=t_{p}$, both cell OCVs and RC pair voltages cannot change instantaneously according to (1) and (11). Thus, any cell OCV imbalance during the series operation will be reserved after the reconfiguration. However, the already equalized RC pair voltages will not contribute to the initial balancing current at $t=t_{p}$, and, thus, can be ignored for estimating the maximum initial switch current. This can be verified in Fig. 3. When RC pairs are removed from the circuit diagram in Fig. 2 (b), i.e., assuming $V_{n}^{m}(t)=0$, the simulated switch currents (marked by dotted curves) start with the same initial currents as those simulated using the complete circuit model including RC pairs (marked by solid curves).

To analyze the switch current between any two adjacent cells at any time instant of the parallel operation without external charging/discharging current, the system can be viewed as two two-terminal networks connected through parallel switches, as illustrated in Fig. 4. The upper branch represents the network consisting of the first to the $m$-th parallelconnected cells in Fig. 2 (b), and the lower branch represents the network including all remaining parallel-connected cells. The two networks are connected through a pair of parallel switches. The Thevenin equivalent circuit parameters of the upper network can be directly calculated by the following proposition, and those of the lower network can be similarly obtained by counting the cells backward.

Proposition 1: At the beginning of the parallel operation in Fig. 2 (b), given a zero total external current, zero RC pair voltages, and identical parameter values as specified in (10), the network composed of the first to the $m$-th parallel-connected cells can be represented by an equivalent voltage source with voltage $V_{e}^{m}$ connected in series with an equivalent resistor with resistance $R_{e}^{m}$. These parameters can be calculated by

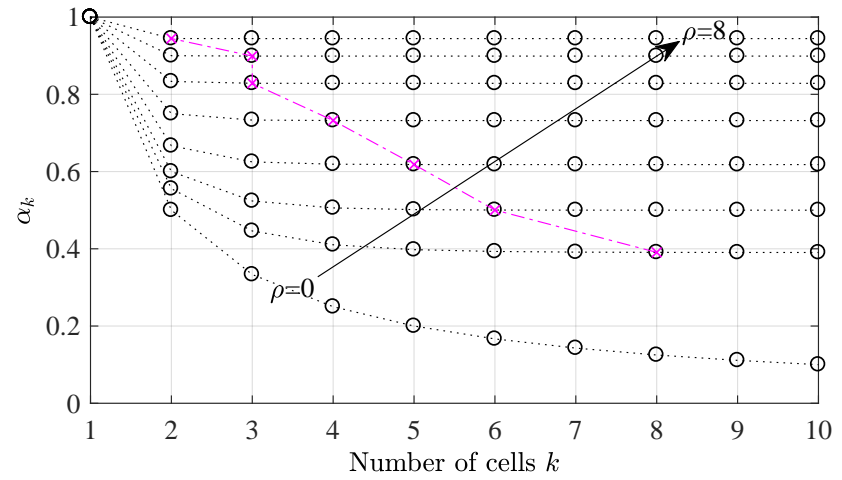

Fig. 5. Evolution of $\alpha_{k}$ with various switch-battery resistance ratios, $\rho \in$ $\left\{0, \frac{1}{8}, \frac{1}{4}, \frac{1}{2}, 1,2,4,8\right\}$, and the arrow indicates the ascending direction of $\rho$.

$R_{e}^{m}=\alpha_{m} R_{C}$

$V_{e}^{m}=$

$$
\left\{\begin{array}{l}
V_{O C}^{1}, \text { if } m=1 \\
\sum_{k=1}^{m-1}\left(\prod_{j=k+1}^{m}\left(1-\alpha_{j}\right) \alpha_{k} V_{O C}^{k}\right)+\alpha_{m} V_{O C}^{m}, \text { if } m>1
\end{array}\right.
$$

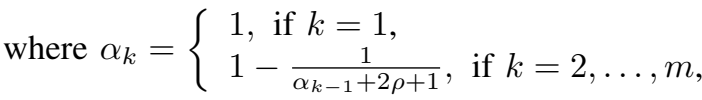

$$
\rho=R_{S} / R_{C} \text {. }
$$

1) Analysis of the equivalent network resistance $R_{e}^{m}$ : It can be seen from (17) that $R_{e}^{m}$ depends on the $\alpha_{m}$ which can be obtained according to the $\alpha_{k}$ sequence defined in (19) and relying only on the switch-battery resistance ratio $\rho$ defined in (20). Given different resistance ratios, the $\alpha_{k}$ sequences are compared in Fig. 5. For each $\rho, \alpha_{k}$ decreases and eventually approaches convergence as the number of cells involved, i.e., $k$, increases. Given a larger $\rho, \alpha_{k}$ can converge to a higher value involving a smaller number of cells $k$ (marked by magenta cross points). Here, a convergence tolerance $\epsilon_{c}=10^{-3}$ is set. These observations can be mathematically proved and generalized in Lemma 1.

Lemma 1: Given $\rho \geq 0$ in the sequence of $\alpha_{k}$ defined in (19), as the number of involved cells $k$ increases from $1, \alpha_{k}$ monotonically decreases from 1 and finally converges to

$$
\alpha_{\infty}(\rho) \triangleq \lim _{k \rightarrow+\infty} \alpha_{k}(\rho)=\sqrt{\rho^{2}+2 \rho}-\rho .
$$

Note that, the fast convergence property of $\alpha_{k}$ as seen in Fig. 5 can be applied to further simplify the calculation of the equivalent resistance $R_{e}^{m}$. Given $\rho>0$ and the convergence tolerance $\epsilon_{c}$, let $k_{c}\left(\rho, \epsilon_{c}\right)$ denote the smallest number of cells $k$ such that $\alpha_{k}(\rho)-\alpha_{\infty}(\rho) \leq \epsilon_{c}$. Then, $\alpha_{k}(\rho) \approx \alpha_{\infty}(\rho)$, $\forall k \geq k_{c}$. Thus, according to (17), the equivalent resistance of any network including at least $k_{c}$ cells can be approximated by a constant resistance, i.e.,

$$
R_{e}^{m} \approx \alpha_{\infty}(\rho) R_{C}, m \geq k_{c}\left(\rho, \epsilon_{c}\right), \rho>0 .
$$

2) Analysis of the equivalent network voltage $V_{e}^{m}$ : Unlike the equivalent resistance only dependent on the sequence of $\alpha_{k}$, according to (18), the equivalent voltage $V_{e}^{m}$ is also influenced by the OCVs of all cells involved. The equivalent voltage $V_{e}^{m}$ can be viewed as a weighted sum of all $m$ OCVs 
and it can be proved that all weights fall within $\in(0,1]$ and sum up to one. Then,

$$
V_{e}^{m} \in\left[\min _{1 \leq k \leq m} V_{O C}^{k}, \max _{1 \leq k \leq m} V_{O C}^{k}\right]
$$

Particularly, if all cells have the same initial OCV in a network, i.e., $V_{O C}^{k}=V_{O C}, k=1, \ldots, m$, then, according to (18), the equivalent voltage can be directly obtained as below regardless of the number of cells involved $k$ and the resistance ratio $\rho$.

$$
V_{e}^{m}\left(V_{O C}^{k}=V_{O C}, k=1, \ldots, m\right)=V_{O C}
$$

3) Switch current expression based on the network model: According to the equivalent circuit in Fig. 4, (10), (17), and (20), the initial magnitude of the $m$-th switch current is

$$
\left|I_{S z i}^{m}\right|=\frac{\left|V_{e}^{m}-V_{e}^{M-m}\right|}{R_{e}^{m}+R_{e}^{M-m}+2 R_{S}}=\frac{\left|V_{e}^{m}-V_{e}^{M-m}\right|}{\left(\alpha_{m}+\alpha_{M-m}+2 \rho\right) R_{C}} .
$$

Given the resistances of batteries and switches, the denominator in (25) can be evaluated based on (19) and (20), but the numerator still depends on the cell OCVs at the moment of the system reconfiguration according to (18). Thus, the switch currents under various cell OCVs are then analyzed.

\section{Estimating the maximum switch current under various cell OCVs without total charging/discharging current}

When reconfiguring the battery connection from series to parallel, battery OCVs could differ from each other and severe battery OCV imbalance could cause a series of progressive issues, such as biased current and thermal distributions, higher total power loss, reduced energy storage/delivery, and even safety hazards [21]. All battery OCVs and SoCs can be estimated using well-designed state estimators [22]-[24], and they do not change instantaneously during the system reconfiguration. Thus, a limit is commonly imposed on the range of all battery OCVs to restrict the unbalanced operation. Based on (23), the numerator of $\left|I_{S z i}^{m}\right|$ in (25)

$$
\left|V_{e}^{m}-V_{e}^{M-m}\right| \leq V_{O C}^{\text {ran }} \leq V_{O C}^{r l i m},
$$

where $V_{O C}^{r a n} \triangleq \max _{1 \leq k \leq M} V_{O C}^{k}-\min _{1 \leq k \leq M} V_{O C}^{k}$ is the range of all $M$ battery OCVs and $V_{O C}^{r l i m}$ is its upper limit. Particularly, according to (24), the equality in (26) can be achieved at some $m=m^{*}$, if $V_{O C}^{1}=\ldots=V_{O C}^{m^{*}}, V_{O C}^{m^{*}+1}=\ldots=V_{O C}^{M}$, and $\left|V_{O C}^{1}-V_{O C}^{M}\right|=V_{O C}^{\text {rlim }}$.

Moreover, given the resistances of batteries and switches, the switch-battery ratio $\rho$ can be determined following (20), and the sequences of $\alpha_{m}$ and $\alpha_{M-m}$ can be calculated based on (19). Then, there exists some $m^{\dagger}$ at which the sum of $\alpha_{m}$ and $\alpha_{M-m}$, denoted by $S_{\alpha}^{m}(\rho, M)$, reaches its minimum, i.e.,

$$
S_{\alpha}^{m^{\dagger}}(\rho, M)=\alpha_{m^{\dagger}}+\alpha_{M-m^{\dagger}} \triangleq \min _{1 \leq m \leq M-1}\left(\alpha_{m}+\alpha_{M-m}\right) .
$$

It can be shown that the $\alpha_{k}$ sequence defined by (19) is convex. Then, $m^{\dagger}=\left\lfloor\frac{M}{2}\right\rfloor$, and

$$
S_{\alpha}^{m^{\dagger}}(\rho, M)=\alpha_{\left\lfloor\frac{M}{2}\right\rfloor}+\alpha_{M-\left\lfloor\frac{M}{2}\right\rfloor}=\alpha_{\left\lfloor\frac{M}{2}\right\rfloor}+\alpha_{\left\lceil\frac{M}{2}\right\rceil} .
$$

Based on (19), (21), (22), (27), (28), and Lemma 1,

$$
\begin{aligned}
& S_{\alpha}^{m^{\dagger}}(\rho, M) \geq 2\left(\sqrt{\rho^{2}+2 \rho}-\rho\right), M>1, \\
& S_{\alpha}^{m^{\dagger}}(\rho, M)= \begin{cases}2, & \text { if } M=2, \\
2-\frac{1}{2(\rho+1)}, & \text { if } M=3,\end{cases} \\
& S_{\alpha}^{m^{\dagger}}(\rho, M) \approx 2\left(\sqrt{\rho^{2}+2 \rho}-\rho\right), \text { if } M \geq 2 k_{c}\left(\rho, \epsilon_{c}\right) .
\end{aligned}
$$

Accordingly, the denominator in (25) can be minimized to $\left(S_{\alpha}^{m^{\dagger}}(\rho, M)+2 \rho\right) R_{C}$.

In order to maximize the switch current in (25), the numerator is maximized and the denominator is minimized simultaneously, i.e., letting $m=m^{*}=m^{\dagger}$. Thus, based on the (25), (26), and (27), the maximum switch current magnitude in (16) under various cell OCVs is

$$
\max _{m}\left|I_{S z i}^{m}\left(t_{p}\right)\right| \leq \frac{V_{O C}^{r l i m}}{\left(S_{\alpha}^{m^{\dagger}}(\rho, M)+2 \rho\right) R_{C}} \triangleq\left|I_{S z i}^{\max }\right| .
$$

Then, based on (29), (30), (31), and (32), the maximum switch current magnitudes for different numbers of parallel-connected battery cells can be evaluated by

$$
\begin{aligned}
& \left|I_{S z i}^{\max }(M>1)\right| \leq\left|I_{S z i}^{u l i m}\right|, \\
& \left|I_{S z i}^{u l i m}\right| \triangleq \lim _{M \rightarrow+\infty}\left|I_{S z i}^{\max }\right|=\frac{V_{O C}^{\text {rlim }}}{2 \sqrt{\rho^{2}+2 \rho} R_{C}}=\frac{V_{O C}^{r l i m}}{2 \sqrt{1+\frac{2}{\rho}} R_{S}},
\end{aligned}
$$

$$
\begin{aligned}
& \left|I_{S z i}^{\max }(M=2)\right|=\frac{V_{O C}^{r l i m}}{2(\rho+1) R_{C}}, \\
& \left|I_{S z i}^{\max }(M=3)\right|=\frac{V_{O C}^{r l i m}}{\left(2(\rho+1)-\frac{1}{2(\rho+1)}\right) R_{C}}, \\
& \left|I_{S z i}^{\max }\left(M \geq 2 k_{c}\right)\right| \approx\left|I_{S z i}^{u l i m}\right| .
\end{aligned}
$$

According to (33), the maximum parallel switch currents all share a common upper limit $\left|I_{S z i}^{u l i m}\right|$, defined in (34) and independent of the number of parallel-connected battery cells $M$. For a small number of cells, the upper limits can be directly derived based on (28) and (32), e.g., (35) and (36). When the number of cells $M \geq 2 k_{c}, S_{\alpha}^{m^{\dagger}}(\rho, M)$ approaches its minimum according to (31) and accordingly the maximum switch current approaches its upper limit, as given in (37).

\section{Estimating the maximum switch current during recon- figuration considering the external charging/discharging current}

Finally, taking into account both the voltage balancing between cells of various OCVs and the dynamic distribution of the wide-range external current to parallel cells, the maximum switch current magnitude $\left|I_{S}^{\max }\right|$ and its upper limit $\left|I_{S}^{u l i m}\right|$ can be derived based on (14), (15), (16), (32), (33), and (34),

$$
\begin{aligned}
\max _{m, t}\left|I_{S}^{m}(t)\right| & \leq\left|I_{S}^{\max }\right| \leq\left|I_{S}^{u l i m}\right| \\
\left|I_{S}^{\max }\right| & \triangleq\left|I_{S z i}^{\max }\right|+\left|I_{S z s}^{\max }\right| \\
& =\frac{V_{O C}^{r l i m}}{\left(S_{\alpha}^{m^{\dagger}}(\rho, M)+2 \rho\right) R_{C}}+\max _{m}\left|D_{S}^{m}\right| I_{T}^{u b}
\end{aligned}
$$




$$
\begin{aligned}
\left|I_{S}^{u l i m}\right| & \triangleq \lim _{M \rightarrow+\infty}\left|I_{S}^{\max }\right| \\
& =\frac{V_{O C}^{r l i m}}{2 \sqrt{\rho^{2}+2 \rho} R_{C}}+\max _{m}\left|D_{S}^{m}\right| I_{T}^{u b} \\
& =\frac{V_{O C}^{r l i m}}{2 \sqrt{1+\frac{2}{\rho}} R_{S}}+\max _{m}\left|D_{S}^{m}\right| I_{T}^{u b} .
\end{aligned}
$$

To estimate the switch current upper limit, these analytical expressions, covering all possible battery states and total charging/discharging currents, are much more computationally efficient than performing a large number of circuit experiments and numerical simulations. While (40) yields a higher, and, hence, more conservative estimate than (39), it can quickly provide a reliable estimate without evaluating $S_{\alpha}^{m^{\dagger}}$, especially when a large number of parallel-connected cells are involved.

It is worth noting that, only two parameter values are required when applying these analytical expressions, mainly the battery internal resistance and the switch on-resistance. For new cells and switches, these parameter values can be easily looked up from the datasheets. As battery cells age over time, the ratio $\rho$ decreases since the battery internal resistance $R_{C}$ generally increases [25] and the switch resistance $R_{S}$ almost stays the same. According to the last line in (40), battery degradation contributes to a decreased upper limit of the maximum switch current. Then, the initially selected switches according to the current limit estimated based on the resistance of new battery cells, do not have to upgrade anymore due to the battery degradation. In addition, the battery cell internal resistance [26] The heterogeneous degradation of battery cells does not satisfy the assumption in (10) and will be separately investigated in our future work.

\section{VALIDATION OF THE ACCURACY AND EFFECTIVENESS}

\section{A. Circuit implementation based on $\mathrm{H}$-bridge modules}

To test the performance of the proposed estimation method, a laboratory prototype of the RBS design in Fig. 1 was built as shown in Fig. 6. In this prototype, LG 186502800 mAh battery cells [27] and H-bridges based on low-voltage MOSFETs [28] were used. In addition, automotive fuses rated for $20 \mathrm{~A}$ are attached for current protection.

To illustrate the operation of this RBS, the schematic of this prototype is shown in Fig. 7 (a). Specifically, the switches of each H-bridge are operated in pairs, and the switches of each half-bridge must be complementarily operated. As shown in Fig. 7 (b), both switches $S_{k 4}$ and $S_{(k+1) 1}, 1 \leq k<n$, are conducted in these H-bridge modules for parallel battery operation, while the remaining switches $S_{k 3}$ and $S_{(k+1) 2}$ for series connection. Fig. 7 (c) depicts the logic of the gate signals to activate the parallel or the series connections of all batteries.

Based on the battery and MOSFET switch datasheets and experimental measurements at room temperature $20^{\circ} \mathrm{C}$, the following parameter values are extracted for estimating the maximum switch current. The total cell resistance is $R_{C}=$

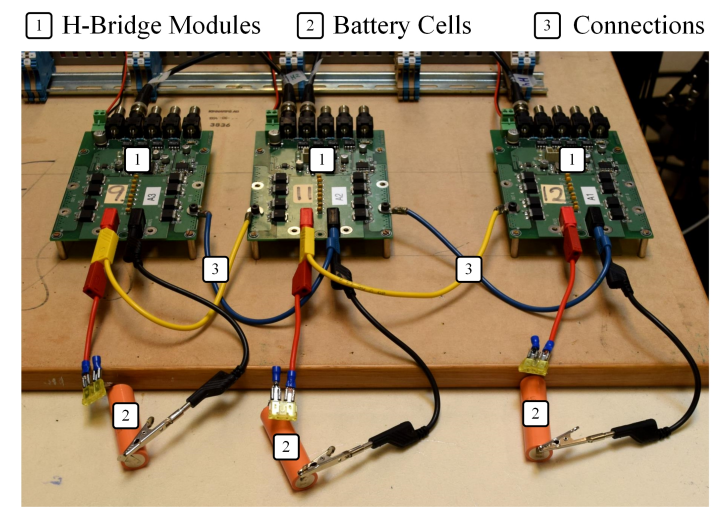

Fig. 6. Laboratory setup with three H-bridge modules.

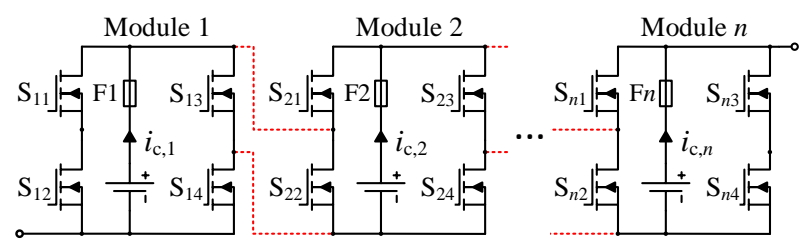

(a)

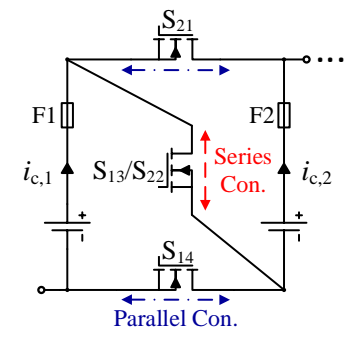

(b)

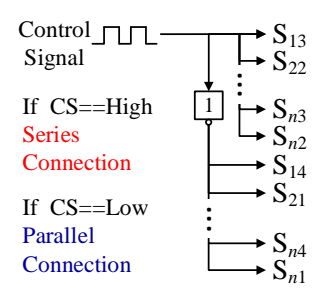

(c)
Fig. 7. (a) Schematic of the RBS implementation based on H-bridge modules. (b) Switches for series and parallel connections. (c) Gate signals for series and parallel connections.

$71.5 \mathrm{~m} \Omega$, composed of the battery's internal resistance $R_{0}=$ $62.1 \mathrm{~m} \Omega$, the connection wire's resistance $6 \mathrm{~m} \Omega$, and the fuse's resistance $3.4 \mathrm{~m} \Omega$. Besides, the parallel switch resistance is $R_{S}=5.3 \mathrm{~m} \Omega$, consisting of the switch on-state resistance $1 \mathrm{~m} \Omega$ and all the resistance along the conduction path on the printed circuit board. Then, based on (20), the switch-battery resistance ratio is $\rho=0.0741$.

\section{B. Circuit experiments for evaluating the performance of the proposed estimation method}

As shown in Section III, the major breakthrough in this paper is deriving the analytical expressions for the maximum switch current and its upper limit during the series-toparallel reconfiguration without external current. To evaluate the performance of the proposed method, circuit experiments were performed using the RBS prototype in Fig. 6. In all the following tests, battery cells, originally connected in series, were reconfigured to a parallel connection at $t_{p}=0$ without external charging/discharging currents.

1) Two-cell test: Two cells with a large initial OCV difference of $1.1 \mathrm{~V}$ were first tested. As shown in Fig. 8, when these cells are reconfigured from series to parallel, the switch current (marked by blue dots) increases rapidly and reaches its peak 7.195 A. During this ascending period, while some 

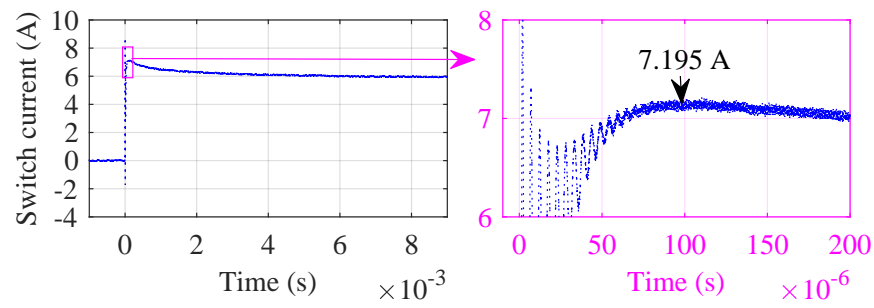

Fig. 8. Experimental switch currents during the series-to-parallel reconfiguration of a two-cell system with initial cell OCVs $(4.17 \mathrm{~V}, 3.07 \mathrm{~V})$.

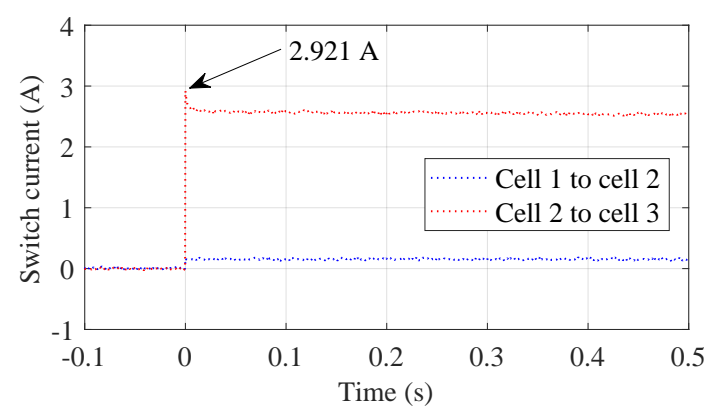

Fig. 9. Experimental switch currents during the series-to-parallel reconfiguration of a three-cell system with initial OCVs $(3.969 \mathrm{~V}, 4.166 \mathrm{~V}$, $3.717 \mathrm{~V})$.

oscillations can be observed due to the resonance of the parasitic inductance and capacitance on associated elements, their magnitudes quickly diminish within microseconds and cannot cause significant damage to switches (e.g., the current limit is imposed to a 160-microsecond pulse in [10]). After passing the peak, the switch current decreases at a much slower pace than the previous ascending period. Thus, only the switch current peak will be analyzed.

Based on the extracted parameter values, the maximum switch current $I_{S}^{\max }$ can be estimated using (35) given the OCV range limit $V_{O C}^{\text {rlim }}$. To facilitate the comparison, set $V_{O C}^{r l i m}$ to the given $\mathrm{OCV}$ range, i.e., $1.1 \mathrm{~V}$. Then, $I_{S}^{\max }$ is estimated to be $7.161 \mathrm{~A}$, very close to the measurement 7.195 A with a percentage error of $-0.47 \%$, which verifies the estimation accuracy of the proposed method.

2) Three-cell test: To demonstrate the effectiveness and advantage of the proposed method, the experimental test was then extended to a three-cell system. It is aimed to estimate the maximum switch current among all possible cell OCV permutations within a specific cell OCV range limit to ensure the safe operation of switches. It would be too inefficient to exhaustively test all possible OCV permutations by experiments or model-based circuit analysis. Thus, one cell OCV permutation $(3.969 \mathrm{~V}, 4.166 \mathrm{~V}, 3.717 \mathrm{~V})$ was randomly selected and experimentally tested. The measured maximum switch current was $2.921 \mathrm{~A}$ as illustrated in Fig. 9.

The maximum switch current for this specific cell OCV permutation can also be evaluated through circuit analysis based on the circuit diagram in Fig. 2 (b) without RC pairs along with the parameter values identified in Section IV-A. The calculated maximum switch current, $2.970 \mathrm{~A}$, is close to the above experimental measurement with a small percentage error of $1.66 \%$, verifying the accuracy of these parameter values and the feasibility of circuit analysis-based current evaluation. Then, using a similar analysis procedure for each permutation

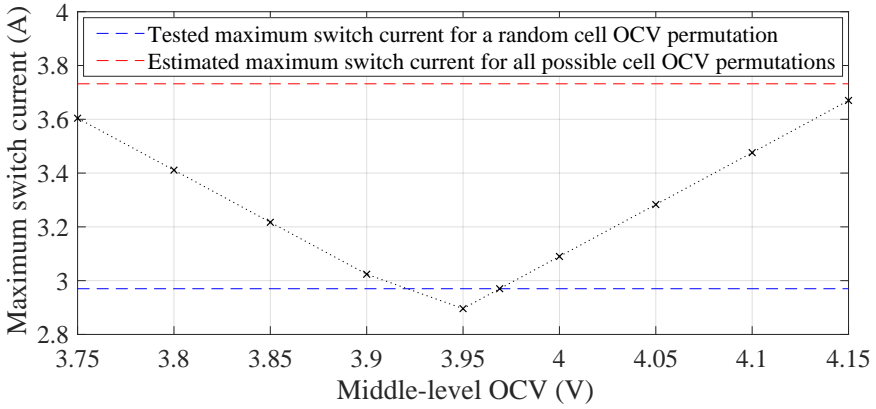

Fig. 10. Maximum switch currents during the series-to-parallel reconfiguration for the same highest cell $\mathrm{OCV}, 4.166 \mathrm{~V}$, the same lowest cell OCV, $3.717 \mathrm{~V}$, but various middle-level cell OCVs.

of these cell OCVs, we can obtain three different maximum switch currents, i.e., $2.970 \mathrm{~A}, 2.757 \mathrm{~A}$, and $2.857 \mathrm{~A}$, since any OCV permutation and its inverse lead to the same result. Thus, the experimentally tested cell OCV permutation happens to yield the maximum switch current of all six possible permutations of the three cell OCVs.

To facilitate the comparison of the proposed switch current estimation method with the above experimental test and circuit analysis, the cell OCV range limit in the proposed method is specified to the range of these three cell OCVs, i.e., $V_{O C}^{r l i m}=$ $0.449 \mathrm{~V}$. However, various cell OCVs and permutations are possible during the system operation, including the above randomly picked cell OCV set tested by the experiment and circuit analysis. Even though the highest $\mathrm{OCV}, 4.166 \mathrm{~V}$, and the lowest cell OCV, $3.717 \mathrm{~V}$, are further fixed, the remaining middle-level cell OCV still varies in between. Then the maximum switch currents for various samples of middle-level cell OCVs are calculated through circuit analysis, as marked by black crosses in Fig. 10. It can be seen that some sampled maximum switch currents are above the tested one for the previous randomly selected cell OCV set (marked by the blue dash line), but all sampled currents fall below the estimated maximum switch current considering all possible cell OCV permutations, i.e., 3.732 A obtained by (36) and marked by the red dash line in Fig. 10. This verifies the effectiveness of the proposed method in terms of providing a reliable estimate of the maximum switch current for various possible operating scenarios. To obtain a reliable estimate, the proposed method outperforms time-consuming experimental tests or circuit analysis of various possible cell OCV permutations.

\section{Estimating the maximum switch currents for larger systems}

After experimentally verifying the accuracy and effectiveness of the proposed method, this method is deployed to assess the maximum switch currents in RBSs larger than the prototype system in Fig. 6. Given a limit of the battery cell OCV range, e.g., $V_{O C}^{r l i m}=0.3 \mathrm{~V}$, based on the battery and switch parameters extracted in Subsection IV-A, i.e., $R_{C}=$ $71.5 \mathrm{~m} \Omega$ and $R_{S}=5.3 \mathrm{~m} \Omega$, the maximum switch currents for RBSs composed of different numbers of cells are estimated using (19), (20), (28), and (32), and demonstrated in Fig.11. The maximum switch current increases as more cells get involved, and approaches its upper limit estimated by (34) 


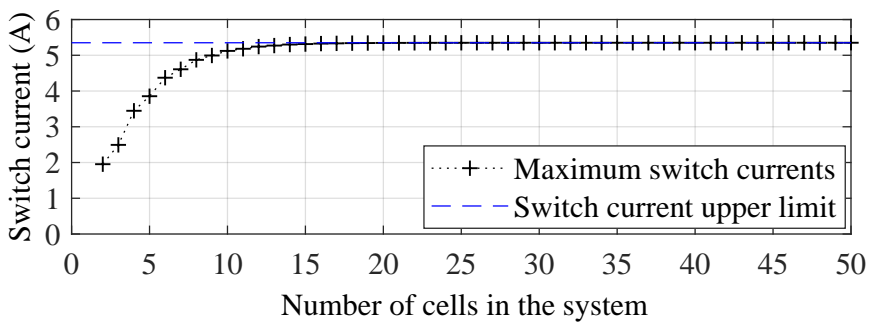

Fig. 11. The maximum switch currents and their upper limit as the number of cells increases in the system.

when the system has more than 12 cells. Thus, for large-scale systems, we only need to estimate the upper limit using (34) and it is reliable to directly view it as the system's maximum switch current regardless of the exact number of cells involved.

The maximum switch current is estimated mainly for the switch current rating during the initial transient period after the system reconfiguration. When it is applied to the switch current rating during the steady-state period, the switch's steady-state current is overrated to some extent, as shown in Fig. 8 and Fig. 9, but it leaves a margin above the actual operating limit, contributing to the reliable system operation.

\section{CONCLUSIONS AND FUTURE WORK}

Flexible system reconfiguration through switch operations provides a new option for battery system management and control. On the other hand, if switches are not properly selected in an RBS, some desired system configurations may become inefficient and even infeasible. This work first investigates the switch currents during battery system reconfiguration for selecting appropriate RBS switches. Specifically, the maximum switch current as well as its upper limit during the mutual conversion between series and parallel connections have been analyzed and estimated considering various possible battery voltages. An RBS prototype was set up and circuit experiments were carried out to demonstrate the accuracy and effectiveness of the proposed estimation method.

As compared to testing a large number of possible cell OCVs and permutations by circuit experiments or modelbased numerical simulations, the proposed estimation method, presented in analytical expressions without massive search, is much more efficient in both energy and time. In addition, while focusing on the reconfiguration between basic series and parallel connections, this work provides a framework to further analyze more complex reconfigurations involving mixed series and parallel connections.

In future work, more temperature levels will be tested, and the influence of heterogeneous battery temperatures and ages on the maximum switch current will be further investigated. Moreover, following the analysis during the system reconfiguration, the switch current peaks throughout the operating cycle, mainly caused by the dynamic external charging or discharging currents, will be studied.

\section{APPENDIX A NOMENCLATURE}

All abbreviations and denotations are specified as follows.

\begin{tabular}{|c|c|}
\hline RBS & Reconfigurable battery system \\
\hline$m$ & Battery cell index \\
\hline$M$ & The total number of cells in the battery system \\
\hline ECM & Equivalent circuit model \\
\hline OCV & Open circuit voltage \\
\hline$V_{O C}^{m}$ & Open circuit voltage of the $m$-th cell \\
\hline$R_{0}^{m}$ & Internal resistance of the $m$-th cell \\
\hline$N$ & The number of RC pairs in each battery cell ECM \\
\hline$R_{n}^{m}$ & $\begin{array}{l}\text { Resistance of the resistor in the } n \text {-th RC pair in the } m \text {-th } \\
\text { cell's ECM }\end{array}$ \\
\hline$C_{n}^{m}$ & $\begin{array}{l}\text { Capacitance of the capacitor in the } n \text {-th RC pair in the } \\
m \text {-th cell's ECM }\end{array}$ \\
\hline$V_{n}^{m}$ & Voltage of the $n$-th RC pair in the $m$-th cell's ECM \\
\hline$R_{S}^{m}$ & Resistance of the series switch in Fig. 2 \\
\hline$R_{S+}^{m}$ & $\begin{array}{l}\text { Resistance of the parallel switch connecting the positive } \\
\text { battery terminals in Fig. } 2\end{array}$ \\
\hline$R_{S-}^{m}$ & $\begin{array}{l}\text { Resistance of the parallel switch connecting the negative } \\
\text { battery terminals in Fig. } 2\end{array}$ \\
\hline$I_{C}^{m}$ & Current of the $m$-th battery cell, positive for charging \\
\hline$I_{S}^{m}$ & $\begin{array}{l}\text { Current of the } m \text {-th parallel switch connecting the positive } \\
\text { battery terminals }\end{array}$ \\
\hline$I_{T}$ & Current of the battery system, positive for charging \\
\hline$x$ & Battery state vector \\
\hline $\mathrm{SoC}$ & State of charge \\
\hline$z_{m}$ & State of charge of the $m$-th battery cell \\
\hline$Q_{m}$ & Amount of charge of the $m$-th battery cell \\
\hline$k_{V S}\left(z_{m}\right)$ & The slope of the OCV-SoC curve at the cell SoC $z_{m}$ \\
\hline & Battery cel current vector \\
\hline$A_{C}, B_{C}$ & System matrices corresponding to the system input $I_{C}$ \\
\hline KVL & Kirchhoff's voltage law \\
\hline KCL & Kirchhoff's current law \\
\hline$E, F, G$ & $\begin{array}{l}\text { Matrices relating the battery state and current vectors to } \\
\text { the battery system current }\end{array}$ \\
\hline$A, B$ & System matrices corresponding to the system input $I_{T}$ \\
\hline$I_{S}$ & Switch current vector \\
\hline$T_{S C}$ & $\begin{array}{l}\text { The Transition matrix from the cell current vector } I_{C} \text { to } \\
\text { the switch current vector } I_{S}\end{array}$ \\
\hline$C_{S}, D_{S}$ & Output matrices corresponding to the output $I_{S}$ \\
\hline$R_{C}$ & The identical internal resistance for all battery cells \\
\hline$R_{n}$ & $\begin{array}{l}\text { The identical resistance of the resistor in the } n \text {-th RC pair } \\
\text { for all battery cells }\end{array}$ \\
\hline$C_{n}$ & $\begin{array}{l}\text { The identical capacitance of the capacitor in the } n \text {-th RC } \\
\text { pair for all battery cells }\end{array}$ \\
\hline$\tau_{n}$ & Time constant of the $n$-th RC pair for all battery cells \\
\hline$R_{S}$ & The identical resistance for all switches \\
\hline$I_{S z i}$ & The switch current given zero system current, i.e., $I_{T}=0$ \\
\hline$I_{S z s}$ & The switch current given zero system states, i.e., $x=0$ \\
\hline$D_{S}^{m}$ & The $m$-th entry in the output vector $D_{S}$ \\
\hline & Upper bound of the system current magnitude $\left|I_{T}\right|$ \\
\hline$\left|I_{S z s}^{\max }\right|$ & $\begin{array}{l}\text { The maximum switch current magnitude given zero system } \\
\text { states, i.e., } x=0\end{array}$ \\
\hline$t_{p}$ & $\begin{array}{l}\text { The time instant at which the system is reconfigured to } \\
\text { the parallel operation }\end{array}$ \\
\hline$R_{e}^{m}$ & $\begin{array}{l}\text { The equivalent resistance for the network composed of the } \\
\text { first m parallel-connected cells }\end{array}$ \\
\hline$V_{e}^{m}$ & $\begin{array}{l}\text { The equivalent voltage for the network composed of the } \\
\text { first m parallel-connected cells }\end{array}$ \\
\hline$\left\{\alpha_{k}\right\}$ & $\begin{array}{l}\text { A sequence derived for calculating the equivalent resis- } \\
\text { tance } R_{e}^{m} \text { and the equivalent voltage } V_{e}^{m}\end{array}$ \\
\hline$\rho$ & $\begin{array}{l}\text { The ratio between the switch resistance and the battery } \\
\text { internal resistance }\end{array}$ \\
\hline & Convergence tolerance for the sequence $\left\{\alpha_{k}\right\}$ \\
\hline$k_{c}($ & $\begin{array}{l}\text { The smallest number of cells at which the sequence }\left\{\alpha_{k}\right\} \\
\text { converges given a convergence tolerance } \epsilon_{c}\end{array}$ \\
\hline$V_{O C}^{r a n}$ & Range of all $M$ battery OCVs \\
\hline & Upper limit of all $M$ battery OCVs' range \\
\hline$m^{*}$ & $\begin{array}{l}\text { The battery cell index at which the equality can be } \\
\text { achieved in Equation (26) }\end{array}$ \\
\hline$S_{\alpha}^{m}(\rho, M)$ & $\begin{array}{l}\text { The sum of } \alpha_{m} \text { and } \alpha_{M-m} \text { given the resistance ratio } \rho \\
\text { and the number of battery cells } M\end{array}$ \\
\hline$m^{\dagger}$ & $\begin{array}{l}\text { The } m \text { at which } S_{\alpha}^{m}(\rho, M) \text { is minimized given the } \\
\text { resistance ratio } \rho \text { and the number of batteries } M\end{array}$ \\
\hline$\left|I_{S z i}^{\max }\right|$ & $\begin{array}{l}\text { The maximum switch current magnitude given zero system } \\
\text { current, i.e., } I_{T}=0\end{array}$ \\
\hline
\end{tabular}




\begin{tabular}{ll}
\hline$\left|I_{S z i}^{\text {ulim }}\right|$ & The upper limit of the switch current magnitude given zero \\
$\left|\boldsymbol{I}_{\boldsymbol{S}}^{\boldsymbol{m a x}}\right|$ & system current, i.e., $I_{T}=0$ \\
$\left|\boldsymbol{I}_{\boldsymbol{S}}^{\text {ulim }}\right|$ & The maximum switch current magnitude \\
\hline
\end{tabular}

\section{REFERENCES}

[1] W. Han, T. Wik, A. Kersten, G. Dong, and C. Zou, "Next-Generation Battery Management Systems: Dynamic Reconfiguration," IEEE Ind. Electron. Magazine, vol. 14, DOI 10.1109/MIE.2020.3002486, no. 4, Dec. 2020.

[2] T. Kim, W. Qiao, and L. Qu, "Power electronics-enabled self-X multicell batteries: A design toward smart batteries," IEEE Trans. Power Electron., vol. 27, DOI 10.1109/TPEL.2012.2183618, no. 11, pp. 4723-4733, Nov. 2012.

[3] W. Han and L. Zhang, "Battery cell reconfiguration to expedite charge equalization in series-connected battery systems," IEEE Robot. Autom. Lett., vol. 3, DOI 10.1109/LRA.2017.2728204, no. 1, pp. 22-28, Jan. 2018.

[4] W. Han, C. Zou, L. Zhang, Q. Ouyang, and T. Wik, "Near-fastest battery balancing by cell/module reconfiguration," IEEE Trans. Smart Grid, vol. 10, DOI 10.1109/TSG.2019.2915013, no. 6, pp. 6954-6964, Nov. 2019.

[5] Z. Zhang, Y. Cai, Y. Zhang, D. Gu, and Y. Liu, "A distributed architecture based on microbank modules with self-reconfiguration control to improve the energy efficiency in the battery energy storage system," IEEE Trans. Power Electron., vol. 31, DOI 10.1109/TPEL.2015.2406773, no. 1, pp. 304-317, Jan. 2016.

[6] M. Alahmad, H. Hess, M. Mojarradi, W. West, and J. Whitacre, "Battery switch array system with application for JPL's rechargeable micro-scale batteries," J. Power Sources, vol. 177, DOI 10.1016/j.jpowsour.2007.11.053, no. 2, pp. 566-578, 2008.

[7] A. Badam, R. Chandra, J. Dutra, A. Ferrese, S. Hodges, P. Hu, J. Meinershagen, T. Moscibroda, B. Priyantha, and E. Skiani, "Software defined batteries," in Proc. ACM 25th Symp. Operating Syst. Principles, DOI 10.1145/3007179, pp. 215-229, 2015

[8] S. Ci, N. Lin, and D. Wu, "Reconfigurable Battery Techniques and Systems: A Survey," IEEE Access, vol. 4, DOI 10.1109/ACCESS.2016.2545338, pp. 1175-1189, 2016.

[9] S. Muhammad, M. U. Rafique, S. Li, Z. Shao, Q. Wang, and X. Liu, "Reconfigurable battery systems: A survey on hardware architecture and research challenges," ACM Trans. Des. Autom. Electron. Syst., vol. 24 , DOI 10.1145/3301301, no. 2, Mar. 2019.

[10] Infineon Technologies AG, "Datasheet MOSFET BSP603S2L," https://www.infineon.com/dgd1/Infineon-BSP603S2L-DS-v01_01-en. pdf?fileId=db3a30431a47d73d011a49742afe014f, (Accessed on 06/27/2020)

[11] L. Teschler, "How and when MOSFETs blow up," Power Electronic Tips, Feb. 2016

[12] W. Han and A. Kersten, "Analysis and estimation of the maximum circulating current during the parallel operation of reconfigurable battery systems," in 2020 IEEE Transp. Electrific. Conf. \& Expo. (ITEC), DOI 10.1109/ITEC48692.2020.9161478, pp. 229-234, 2020.

[13] M. Kuder, J. Schneider, A. Kersten, T. Thiringer, R. Eckerle, and T. Weyh, "Battery modular multilevel management (BM3) converter applied at battery cell level for electric vehicles and energy storages," in PCIM Europe digital days 2020; Int. Exhibition Conf. Power Electron., Intell. Motion, Renewable Energy, Energy Manag., pp. 1-8, 2020.

[14] C. Korte, E. Specht, M. Hiller, and S. Goetz, "Efficiency evaluation of MMSPC/CHB topologies for automotive applications," in IEEE 12th Int. Conf. Power Electron. Drive Syst., pp. 324-330, 2017.

[15] A. Kersten, M. Kuder, E. Grunditz, Z. Geng, E. Wikner, T. Thiringer, T. Weyh, and R. Eckerle, "Inverter and battery drive cycle efficiency comparisons of CHB and MMSP traction inverters for electric vehicles," in 21st European Conf. Power Electron. Appl., pp. 1-12, 2019.

[16] Y. Kim, S. Park, Y. Wang, Q. Xie, N. Chang, M. Poncino, and M. Pedram, "Balanced reconfiguration of storage banks in a hybrid electrical energy storage system," in IEEE/ACM Int. Conf. Comput.Aided Design, DOI 10.1109/ICCAD.2011.6105395, pp. 624-631, Nov. 2011.

[17] B. Molenaar, "Reconfigurable battery system for ultra fast charging of industrial electric vehicles," M.S. thesis, Delft University of Technology, Delft, Netherlands, May. 2010.

[18] J. Fu, "Fundamentals of on-resistance in load switches," Texas Instruments, Jun. 2016.
[19] A. Sattar, "Power MOSFET basics," IXYS Corporation, Milpitas, CA, 2007.

[20] "AN-9010 MOSFET basics," Fairchild Semiconductor Corporation, Sunnyvale, CA, 2013. [Online]. Available: https://www.onsemi.cn/pub/ Collateral/AN-9010.pdf.pdf

[21] W. Han, L. Zhang, and Y. Han, "Mathematical modeling, performance analysis and control of battery equalization systems: Review and recent developments," in Advances in Battery Manufacturing, Services, and Management Systems, J. Li, S. Zhou, and Y. Han, Eds., ch. 12, pp. 281-301. New York: Wiley-IEEE Press, 2016.

[22] C. Zou, C. Manzie, D. Nešić, and A. G. Kallapur, "Multi-time-scale observer design for state-of-charge and state-of-health of a lithium-ion battery," J. Power Sources, vol. 335, DOI 10.1016/j.jpowsour.2016.10.040, pp. $121-130,2016$

[23] J. Chen, Q. Ouyang, C. Xu, and H. Su, "Neural network-based state of charge observer design for lithium-ion batteries," IEEE Trans. Control Syst. Technol., vol. 26, DOI 10.1109/TCST.2017.2664726, no. 1, pp. 313-320, 2018.

[24] W. Han, C. Zou, C. Zhou, and L. Zhang, "Estimation of cell SOC evolution and system performance in module-based battery charge equalization systems," IEEE Trans. Smart Grid, vol. 10, DOI 10.1109/TSG.2018.2867017, no. 5, pp. 4717-4728, Sept. 2019.

[25] D. Wang, Y. Bao, and J. Shi, "Online lithium-ion battery internal resistance measurement application in state-of-charge estimation using the Extended Kalman Filter," Energies, vol. 10, DOI 10.3390/en10091284, no. 9, 2017.

[26] A. Kersten, M. Kuder, W. Han, T. Thiringer, A. Lesnicar, T. Weyh, and R. Eckerle, "Online and on-board battery impedance estimation of battery cells, modules or packs in a reconfigurable battery system or multilevel inverter," in 46th Annu. Conf. IEEE Ind. Electron. Soc., DOI 10.1109/IECON43393.2020.9254515, pp. 1884-1891, 2020.

[27] LG Chem, "Product Description: ICR18650 C2 2800 mAh," https://www.batteryspace.com/prod-specs/5702_5.pdf, (Accessed on $07 / 01 / 2019$ ).

[28] Infineon Technologies AG, "Datasheet MOSFET IPB025N10N3G," https://www.infineon.com/dgd1/Infineon-IPB025N10N3G-DS-v02_ 03-en.pdf?fileId=db3a30431ce5fb52011d1ab1d9d51349, (Accessed on $06 / 27 / 2019)$

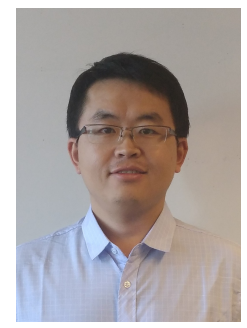

Weiji Han (Member, IEEE) received the B.E. and M.E. degrees from the Department of Electrical Engineering, Shandong University, China, in 2009 and 2012, respectively, and the second M.E. degree and the Ph.D. degree in Electrical Engineering from the Department of Electrical and Computer Engineering, University of Connecticut, Storrs, CT, USA, in 2015 and 2018, respectively.

$\mathrm{He}$ is currently a Researcher in the Department of Electrical Engineering, Chalmers University of Technology, Gothenburg, Sweden. His research interests include modeling, analysis, and control of battery systems with applications to electric vehicles and power grid energy storage. Besides, he also has research experiences in the power system, photovoltaic system, and manufacturing system.

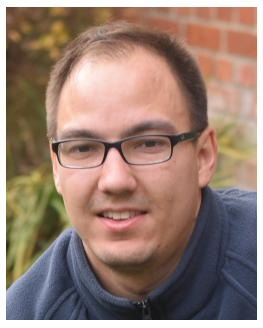

Anton Kersten (Graduate Student Member, IEEE) received his M.Sc. degree in electrical engineering in 2017 from Chalmers University of Technology, Gothenburg, Sweden, where he is currently pursuing his Ph.D. degree. Since 2017, he has been with the Division of Electric Power Engineering, Chalmers University of Technology, Gothenburg, Sweden, where he is involved in the field of multilevel inverters for vehicle powertrains. His research interests include the modeling and control of power electronics for electric drives and the grid integration of renewable energy sources. 


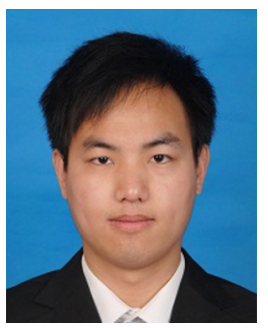

Changfu Zou (Member, IEEE) received the B.E. degree in automotive engineering from the Beijing Institute of Technology, Beijing, China, in 2011 and the Ph.D. degree in automation and control engineering from the University of Melbourne, Melbourne, VIC, Australia, in 2017.

He was a visiting student researcher with the Energy, Controls \& Applications Lab, University of California, Berkeley, Berkeley, CA, USA, from 2015 to 2016. Since 2017, he has joined the Automatic Control Group, Chalmers University of Technology, Gothenburg, Sweden, where he was as a Postdoctoral Researcher and currently is an Assistant Professor. His current research interests include modeling and control of energy storage systems for automotive and power grid applications.

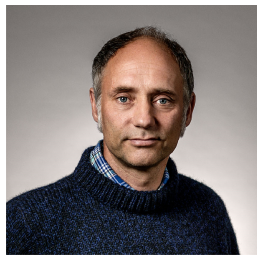

Torsten Wik is a Professor and the head of Automatic Control at the Department of Electrical Engineering, Chalmers University of Technology, Sweden. He received a M.Sc. in Chemical Engineering, a Licentiate of Engineering degree in Control Engineering in 1996, a Ph.D. in Environmental Sciences in 1999 (majoring in Automatic Control), and a Docent degree in 2004, all degrees from Chalmers University of Technology. From 2005 to 2007 he worked as a senior researcher at Volvo Technology in control system design. For the last decade he has led a growing group of researchers on battery management systems.

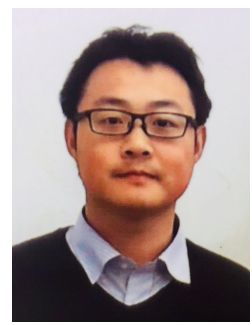

Xiaoliang Huang (Member, IEEE) received the B.S. and M.S. degrees from Jilin University, Changchun, China, in 2007 and 2009, respectively, and the Ph.D. degree from The University of Tokyo, Tokyo, Japan, in 2014.

$\mathrm{He}$ is currently a Researcher with the Department of Electrical Engineering, Chalmers University of Technology, Gothenburg, Sweden. His current research interests include powertrain control, energy storage, and wireless power transfer for electric vehicles.

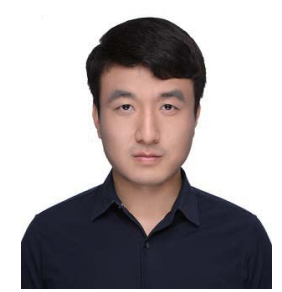

Guangzhong Dong (Member, IEEE) received the B.Eng. degree in automation and the Ph.D degree in control science and engineering from the University of Science and Technology of China (USTC), Hefei, China, in 2013 and 2018, respectively. He was a Postdoctoral Fellow with the School of Data Science, City University of Hong Kong, Hong Kong from 2018 to 2019. $\mathrm{He}$ is currently a Postdoctoral Fellow with the Department of Electrical Engineering, Chalmers University of Technology, Gothenburg, Sweden. His current research interests include dynamic system modeling and control, such as modeling, estimation, health prognosis of energy storage system, optimal energy dispatch and coordinated control of micro-grids.

Dr. Dong was a recipient of the President Prize of Chinese Academy of Sciences for Special Ph.D. Graduates. 